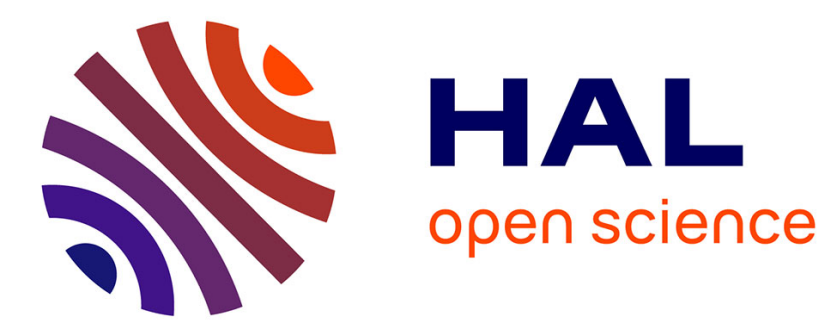

\title{
Photoacoustic molecular spectroscopy and chemical characterization
}

\author{
R. Palmer, Yuchao Jiang
}

\section{To cite this version:}

R. Palmer, Yuchao Jiang. Photoacoustic molecular spectroscopy and chemical characterization. Journal de Physique IV Proceedings, 1994, 04 (C7), pp.C7-337-C7-344. 10.1051/jp4:1994780 . jpa00253309

\section{HAL Id: jpa-00253309 https://hal.science/jpa-00253309}

Submitted on 1 Jan 1994

HAL is a multi-disciplinary open access archive for the deposit and dissemination of scientific research documents, whether they are published or not. The documents may come from teaching and research institutions in France or abroad, or from public or private research centers.
L'archive ouverte pluridisciplinaire HAL, est destinée au dépôt et à la diffusion de documents scientifiques de niveau recherche, publiés ou non, émanant des établissements d'enseignement et de recherche français ou étrangers, des laboratoires publics ou privés. 
JOURNAL DE PHYSIQUE IV

Colloque C7, supplément au Journal de Physique III, Volume 4, juillet 1994

\title{
Photoacoustic molecular spectroscopy and chemical characterization
}

\author{
R.A. Palmer and Y. Jiang \\ Department of Chemistry, Duke University, Box 90346, Durham, North Carolina 27708-0346, U.S.A.
}

\begin{abstract}
Applications of photoacoustic/photothermal (PA/PT) detection to molecular spectroscopy are reviewed with special emphasis on the use of step-scan Fourier transform infrared (FT-IR) PA spectroscopy for chemical analysis and for spectral depth profiling of laminar and other heterogeneous materials, particularly by use of the phase of the PA signal. Examples are drawn from recent work in our laboratory, as well as from the work of others, as appropriate.
\end{abstract}

\section{INTRODUCTION}

Starting with the recognition in the early 1980's that the interferometer offered an ideal source for photoacoustic spectroscopy (PAS), interest and research in infrared, particularly molecular vibrational, PAS has blossomed. As in applications to electronic spectroscopy, the particular characteristics of the frequency domain photothermal effect have provided access to infrared spectra unavailable by use of other techniques (for example, strongly absorbing materials, opaque materials, samples in situ, strongly lightscattering samples). More recently, with the added availability of step-scan methods, the wide free spectral range of Fourier transform infrared (FT-IR) has been increasingly applied in conjunction with PA detection for infrared spectral depth profiling of laminar and otherwise optically heterogeneous materials.

\section{BACKGROUND}

\subsection{PA/PT Molecular Spectroscopy}

Chemical, and particularly analytical molecular spectroscopy using photoacoustic and/or photothermal (PA/PT) detection has made important contributions in the investigations of a wide variety of materials, particularly in the optical regions of the electromagnetic spectrum--IR to UV. The extraordinary sensitivity of gas phase PA/PT detection and the low detection limits and sensitivity to non-enthalpic $\Delta V$ non-radiative changes and time resolution capabilities of PA/PT detection in liquids have helped to carve special niches for photothermal methods in these areas of molecular spectroscopy. However, it was the "rediscovery" of the photothermal effect in solids in the mid 1970's that triggered the resurgence of activity in the field in general, and it is probably true that today the majority of PA/PT work is in the area of solid state materials analysis and characterization, including both thermal and spectral applications. While undifferentiated white light and single laser line (but non-specific absorption) studies, usually directed at determination of thermal properties and imaging, have assumed a dominant position in the broader solid state PA/PT field, specific spectrochemical studies continue to find important application. This is, of course, particularly true where the special characteristics of PA/PT detection provide distinctive advantages over other analytical methods. 
The techniques of spectral differentiation coupled with PA/PT detection have included use of filters, [1] dispersive monochromators, [2] tunable lasers[3] and interferometers[4]. Because of the direct relation of PA/PT signal intensity to incident photon flux, dispersive monochromators are at a distinct disadvantage. In the infrared in particular, owing to the weakness of broadband sources and the relative insensitivity of detectors, dispersive techniques have proved virtually useless for PA/PT spectroscopy. Filters provide higher throughput and can be useful in cases where distinctive absorption features are broad (as in the UV-vis-NIR) and only rough discrimination is required. In terms of incident power, lasers are, of course, unmatched for spectral brilliance, but the limited tuning ranges available and high costs have limited their general application, although for selected problems, currently available tunable IR lasers are very promising. (Use of "tunable" $\mathrm{CO}_{2}$ lasers has a long history in gas phase PAS.) In the future, availability of the broadband spectral brilliance of synchrotron radiation and of the broad tunability with high spectral brilliance of the free electron laser will offer exciting prospects for a wide range of molecular spectroscopy using PA/PT detection.

\subsection{Interferometric Methods for PA/PT Molecular Spectroscopy}

The utility of interferometric methods for PA/PT spectroscopy was demonstrated in the early 1980's. [5] The most obvious natural advantage of interferometry over dispersive techniques for PA/PT detection is the relatively high optical throughput. However, a more subtle aspect of the combination is that in a conventional optical interferometer, the intensity modulation necessary to generate the (frequency domain) PA/PT signal is provided intrinsically by the "Fourier" frequencies, thus eliminating need for any other light modulation device such as a chopper. In addition, the other advantages of interferometry over dispersive methods also apply; that is, speed, wide spectral range and high spectral resolution without sacrificing throughput. These advantages have made PA detection, particularly the standard sample-gasmicrophone PAS method, a widely used technique for qualitative Fourier transform inf rared (FT-IR) spectroscopy in recent years. [6]

However, conventional continuous-scan, or rapid-scan, optical interferometry, as exemplified by most standard commercial FT-IR spectrometers, still has three distinct disadvantages for use with PA/PT detection. All are connected to the constant velocity of the change in retardation (usually the constant velocity of the moving mirror). The problem is that the Fourier frequencies $\mathrm{f}(\mathrm{Hz})$ depend on the wavelength $\lambda(\mathrm{cm})(f=2 v / \lambda$, where $v$ is the velocity of the mirror in $\mathrm{cm} / \mathrm{s})$. The first consequence is that for a given velocity the PA/PT signal will become very much weaker at shorter wavelengths, effectively precluding the use of the technique in the visible, for example. The second problem restricting full application of rapid-scan FT-IR to PAS is that the dependence of $f$ on $v$ leads to a necessary dependence of the thermal diffusion depth $\mu$ on $\lambda$. For example, $\mu$ at $25 \mu \mathrm{m}$ will be approximately three times as great as

that at $2.5 \mu \mathrm{m}$ since $\mu \alpha \lambda^{1 / 2}$. Finally, the lack of a discrete modulation frequency makes extraction of the phase of the PA signal difficult. These latter two problems are particularly significant for their effect on the use of FT-IR PAS for spectral depth profiling of heterogeneous materials.

\subsection{Step-Scan FT-IR PAS}

The solution of these problems, as demonstrated in 1980 by Eyring[7] and by Débarre, Boccara and Fournier[8] is to change the retardation incrementally, step-by-step, rather than at constant velocity. By this device the spectral multiplexing of the interferometer is removed from the time domain; there are effectively no Fourier frequencies. A single discrete modulation frequency can be used which applies to all wavelengths. Thus, not only can step-scan interferometry be applied in the UV-Vis-NIR region with reasonable signal strength (by use of a single, low-frequency modulation), but the thermal diffusion length will be constant across the whole spectrum. In addition, the use of a discrete modulation frequency (or several discrete frequencies) allows the use of lock-in amplifier or digital signal processor methods and thus easy extraction of the signal phase. This last advantage derives from the easy simultaneous recording of the in-phase I and in-quadrature $Q$ components of the signal, and thus the elimination of the wavelength-dependent instrument phase in calculation of the PA signal phase $\phi$.

Since $\phi(\lambda)=\tan ^{-1}[Q(\lambda) /[(\lambda)]$, the instrument phase cancels out. [9]

Thus the essence of step-scan interferometry is that the retardation is changed incrementally; data are collected while the retardation is constant (or while it is oscillated about a fixed position); the multiplex, throughput and registration advantages of interferometry over dispersive techniques are all retained; and the spectral multiplexing is removed from the time domain. The resultant power of interferometry for 
PA/PT spectroscopy for UV-Vis-NIR measurements has not been fully exploited since the early work of Boccara and Fournier. [ ] However, in the infrared step-scan FT-IR PAS has become a significant area of analytical spectroscopy, particularly as applied to spectral depth profiling.[10] Although similar depth profiling measurements at shorter wavelengths are also possible, the nature of the absorption bands in the infrared (narrow, moderate intensity, distinctive of molecular structure) makes the prospects for PA spectral clepth profiling intrinsically more promising in the inf rared than in the NIR, visible or UV.

In the application of step-scan FT-IR to PA/PT spectroscopy, the discrete modulation frequency (or frequencies) used to generate the PA/PT signal may be created externally or internally. External modulation, or amplitude modulation (AM), can be created by a simple chopper or a polarizer/photoelastic modulator combination. Internal modulation, also called path difference modulation or phase modulation (PM), is created by modulation of the length of one arm of the interferometer. For reasons pointed out by Chamberlain, [11] PM is much superior to AM for PA/PT spectroscopy. In short, PM produces the derivative of the AM interferogram.

Sitep-scan FT-IR instruments have been used, particularly in far-infrared and astrophysical applications for many years. However, interest in applications of step-scan methods for PA/PT spectroscopy date from 1980.[7][8] The demonstration of the use of the helium-neon laser for optoelectronic feedback control of the position and stepping of the mirror, although originally applied to NIR-Vis: PTS, [8] heightened interest in the development of instrumentation for mid-infrared PAS/PTS. Both commercial and research modifications of commercial FT-IR instruments were introduced in 1987,[12] [13] and further commercial development followed in 1991.[14]

\subsection{Sprectral Depth Profiling by use of Step-Scan FT-IR PAS}

Perhaps the single most distinctive characteristic of PA/PT detection is its capability for nondestructive depth profiling. The range of depth profile determination and the resolution of that determination depend critically on the range of modulation frequencies available. In addition to the instrumental limitations on modulation frequencies, the intrinsically low powered broadband sources available in the mid-IR dictate that modulation frequencies in excess of $1 \mathrm{KHz}$ will produce poor signal to noise. In continuous-scan operation this means the use of low mirror velocities is required. In step-scan operation with phase modulation, the range is further restricted by the power available to drive the modulation at the amplitude required for the efficient modulation of the infrared wavelengths of interest. In general, with currently available instrumentation the range of depth profiling in dielectric materials is on the order of tens of micrometers, with detection extending to perhaps $100 \mu \mathrm{m}$ in favorable cases.

While the thermal probe depth will be smaller with higher modulation frequencies since $\mu \propto \mathrm{f}^{-1 / 2}$, the potential depth resolution should be greater for higher modulation frequencies. This is because the resolution of the signal phase should not depend on the modulation frequency, and as a result, the minimum distinguishable phase difference will represent a smaller increment of depth at the higher frequericy as compared to the lower. Presuming a range of modulation frequencies of 100 to $1000 \mathrm{~Hz}$ and thermal properties typical of non-conducting organic polymers, predicts a range of $\mu$ of from 18 to $6 \mu \mathrm{m}$. Assuming (conservatively) that probing to a depth of $2 \mu$ is possible, that this may produce a phase difference between the PA signal from a surface species and from one buried $2 \mu$ below the surface of $90^{\circ}$, and that the phase can be reliably determined to $\pm 1^{\circ}$, then the resolution at the higher frequency (and more shallow depth) would be $-0.1 \mu \mathrm{m}$ and at the lower frequency, on the order of $0.4 \mu \mathrm{m}$. Of course, material specific factors, such as concentration of target material, layer thickness, dipole strength of distinctive absorption bands, and actual thermal properties, will modify these predictions.

Thus the predicted scope of depth profiling by use of FT-IR PAS is distinctly different from that of potentally competitive techniques. Although it is a surface-oriented technique, it clearly does not appros.ch the sensitivity of high vacuum surface techniques, or even non-vacuum optical reflectionabsorption spectroscopy or attenuated total reflection (ATR) spectroscopy. However, it has the capability of non-destructively characterizing not only surface layers but also, simultaneously, sub-surface components as well, and to probe at least an order of magnitude deeper than even variable angle ATR. Its probe range is tens of micrometers and its depth resolution is on the order of $1 \mu \mathrm{m}$.

These characteristics of FT-IR PA spectral depth profiling make it applicable to a variety of practical characterization problems related to both continuously heterogeneous materials and discretely heterogeneous materials. These include heterogeneous materials with continuously varying composition such as weathered, corroded and chemically modified surfaces, blooming of plasticizers and barrier 
diffusion problems. In the area of discretely heterogeneous materials, laminated packaging materials, painted surfaces, lubricants and other discrete surface films are obvious possibilities.

In PA spectral depth profiling two fundamental aspects of the photothermal effect and of the sample-gas-microphone technique in particular are taken advantage of: the variation of the thermal probe depth with modulation frequency and the dependence of the signal phase in heterogeneous materials on the depth of the absorber beneath the surface. In FT-IR PAS several strategies can be employed, depending on the level of information needed. As an initial test of heterogeneity, spectra can be measured at different mirror velocities (rapid-scan) or different PM frequencies (step-scan) and normalized to PA spectra of a photothermally saturated reference measured at the same frequencies. The behavior of the signals from truly thermally thin surface films is unambiguous in this test. Their normalized intensities will remain insensitive to changing frequency, while bands originating from materials in interior layers and otherwise part of the substrate, or bulk, will decrease in magnitude as the frequency increases. (Strict adherence to this prediction, of course, assumes that the surface remains thermally thin for all frequencies used and that absorption bands in the bulk chosen for comparison are not photothermally saturated.)

Depth profiling beyond the initial observations obtained from varying the modulation frequency requires the use of the phase of the signal. The level of accuracy required to make this useful virtually requires the use of the step-scan mode, in which, as stated above, the instrument phase is completely canceled out. As is the case in the frequency studies, the goal is to select absorption bands for each component which are free of interference from bands in other components. The relatively narrow band widths in the infrared (even in condensed phases) and the wide spectral range available in FT-IR make this a reasonable goal. Particularly in discretely layered samples, the phase of signal at each band maximum should be distinctive for the depth of the associated layer.

In perhaps the simplest application of phase analysis to depth profiling, the Rosenscwaig-Gersho thermal piston model[15] predicts that the phase of signals from a thermally thin weakly absorbing surface layer will differ from that of bands from a thermally thick and weakly absorbing substrate by $45^{\circ}$.[16] This has been clearly demonstrated in several FT-IR PAS studies of laminar organic polymer materials. [10] Separate simultaneous direct detection of the surface and substrate signals is not possible, even with a two phase lock-in amplifier, since the signals are not orthogonal to each other. However, it is a relatively simple matter to determine the experimental phase angle at which a characteristic band associated with a particular layer is maximized and then to set the detector phase angle exactly orthogonal to that so as to effectively null that signal. While nulling signals from one of two layers is simple (provided at least one layer is thermally thin and the bands are not overlapping) and gives clean separation of the spectra of the layers, distinction of more than two layers requires more elaborate treatment. The ideal case is one of strongly absorbing, non-overlapping chromophores in discrete, thermally thin layers with a total sample thickness on the order of 10 to $50 \mu \mathrm{m}$.

In practice it is not necessary to record spectra at each desired phase angle. In fact, it is only necessary to record two orthogonal components of the interferogram (in a single step scan). All desired phase components can then be calculated by simple vector rotation mathematics. Standard sof tware is available which allows easy rotation of the phase to maximize or null any desired feature in the spectrum.

An alternative approach, and one that offers the possibility of finer distinction between components in terms of depth discrimination, is to use the transforms of the recorded orthogonal components of the signal to calculate the magnitude $M$ and relative phase $\phi$ of the spectrum: $M(\lambda)=\left[I(\lambda)^{2}+Q(\lambda)^{2}\right]^{1 / 2}$ and $\phi(\lambda)=\tan ^{-1}[Q(\lambda) / I(\lambda)]$. In regions where the I and $Q$ spectra have values significantly different from zero, that is, in the region of absorption bands in $M(\lambda), \phi(\lambda)$ will represent accurately the relative phase of the signal. In other regions (regions of vanishingly small absorption) $\phi(\lambda)$ will be essentially meaningless. At values of $\lambda$ where coherent values of $\phi(\lambda)$ are calculated, differences in $\phi$ will be specifically and predictably related to the depth of the absorbing species below the surface and to the absorption strength.

As a first approximation, it can be predicted that the phases of absorption bands associated with the same component will be clustered about a value related to the depth distribution of the component, or layer depth, with the relative phase lag associated with weaker bands being greater than that of stronger bands. If, as in the step-scan data reported in this paper, the relative phase $\phi$ can be resolved to at least $\pm 1^{\circ}$, this method provides a distinctly superior depth profile than can be obtained from the spectral phase rotation method described above. In the case of laminated materials the selection of one strong band in the spectrum of each component layer which does not overlap with any absorption in the other layers should provide data in the $\phi$ spectrum indicating clearly the order and relative spacing of the layers. At this point 
quantitative use of this method is best approached empirically by calibration of the relative phase against samples of known composition and structure. However, useful theoretical models would appear to be in the process of development.[17]

\section{EXPERIMENTAL}

The FT-IR PAS data reported here have been obtained at room temperature by use of an IBMBruker IR 44 benchtop FT-IR spectrometer, modified for optional step-scan operation. The intensity modulation of the IR beam is produced by path difference (phase) modulation. This also produces a phase modulation of the HeNe laser which is used in a lock-in feedback loop to control the mirror position and stepping.[18]

An MTEC Model 100 photoacoustic cell with its accompanying preamplifier/power supply was used to obtain all spectra. Helium was used as the transfer gas in the PA cell because of its superior thermal coupling properties and to remove gaseous water and carbon dioxide. The spectrometer was purged with dry air to remove atmospheric $\mathrm{H}_{2} \mathrm{O}$ and $\mathrm{CO}_{2}$. The interferograms were obtained by collecting data at intervals of one wavelength of the reference HeNe laser, to obtain single-sided interferograms of 2048 points, plus 256 phase correction points, to give $8 \mathrm{~cm}^{-1}$ resolution. The in-phase (I) and quadrature (Q) interferograms were collected simultaneously and Fourier transformed after data collection was complete. From the in-phase and quadrature spectra, the magnitude, $M(\lambda)=\left[I(\lambda)^{2}+Q(\lambda)^{2}\right]^{1 / 2}$, and phase, $\phi(\lambda)=\tan ^{-1}[Q(\lambda) / I(\lambda)]$, spectra were calculated. Alternatively, the individual vector components I and $Q$ were used, or components at other angles were calculated as described below.

In order to obtain the instrument throughput magnitude reference and the surface phase angle reference, a $60 \%$ carbon-filled elastomer disk was used. Samples were placed in the cell on a spacer designed to give a constant cell filling factor and to act as a photothermal trap for radiation transmitted by the sample film. All spectra are presented as $S / R$, that is, the sample spectrum divided by the spectrum of the carbon-filled elastomer reference, as is usual in PAS. Reproducibility of the reference phase determination was found to be $\pm 1^{\circ}$ from trial to trial.[10]

For the phase analysis studies, the reference sample was placed in the PA cell to determine the surface reference phase of the lock-in amplifier at each phase modulation (PM) frequency to be used. The reference phase was determined by first stepping the mirror to the zero retardation point, which is the point in the interferogram that contains contributions from all wavelengths. Then the lock-in amplifier phase was rotated until all the signal was located in the quadrature channel. Once this lock-in reference phase was established, the reference was replaced by the sample and the I and Q interferograms were simultaneously recorded.

Transformation of the I and Q interferograms collected as described above gives the I and Q spectra used to calculate $M$ and $\phi$ spectra. In order to obtain vector components at other angles, the $I$ and $Q$

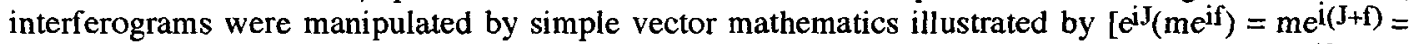
$\operatorname{mcos}(\mathrm{J}+\mathrm{f})+i m \sin (\mathrm{J}+\mathrm{f})]$, where $\mathrm{e}^{\mathrm{i} J}$ is the polar representation of the phase rotation angle $\mathrm{J}$ and $m \mathrm{e}^{\mathrm{if}}$ is the polar representation of a pair of points in the original interferograms (I and Q).

\section{RESULTS AND DISCUSSION}

Figure la shows the step-scan FT-IR PA spectrum of a two layer polymer sample consisting of $12.5 \mu \mathrm{m}$ of ethylene vinyl acetate copolymer (EVAc) on a substrate of $60 \mu \mathrm{m}$ of polypropylene (PP). (The spectra (from the top) are the calculated magnitude, the vector component at a relative phase of $80^{\circ}$, and the component at $115^{\circ}$. Figure $1 \mathrm{~b}$ shows the phase spectrum of EVAc/PP calculated from the same data used in Figure 1a. Note that the EVAc bands identified as A, B and C in Figure 1a, which are maximized in the $115^{\circ}$ component, are associated with relative phase values at their band maxima ranging from $45.5^{\circ}$ to $50.4^{\circ}$, while those bands associated with the PP substrate exhibit $\phi$ values of $66.4^{\circ}, 68.8^{\circ}$ and $83.9^{\circ}$. The difference between the phase associated with the strongest, non-overlapping absorption in the surface layer $(B)$ and that of the weakest, non-overlapping absorption in the substrate $(F)$ is $34.3^{\circ}$. Correspondingly, in Figure 1c the difference in phase angle at which the intensities of these two bands are nulled (or maximized) can be seen to be approximately $35^{\circ}$. The data for the EVAc/PP sample are summarized in Table 1, where the results of the two methods of determining the phase difference between the signals at the absorption band maxima are compared. $\Delta \Phi(\mathrm{spec})$ data are the differences obtained from $\phi(\lambda)$ (Figure 1b), while $\Delta \Phi($ rot) data are obtained from the phase rotation method (Figure Ic) 
(a)

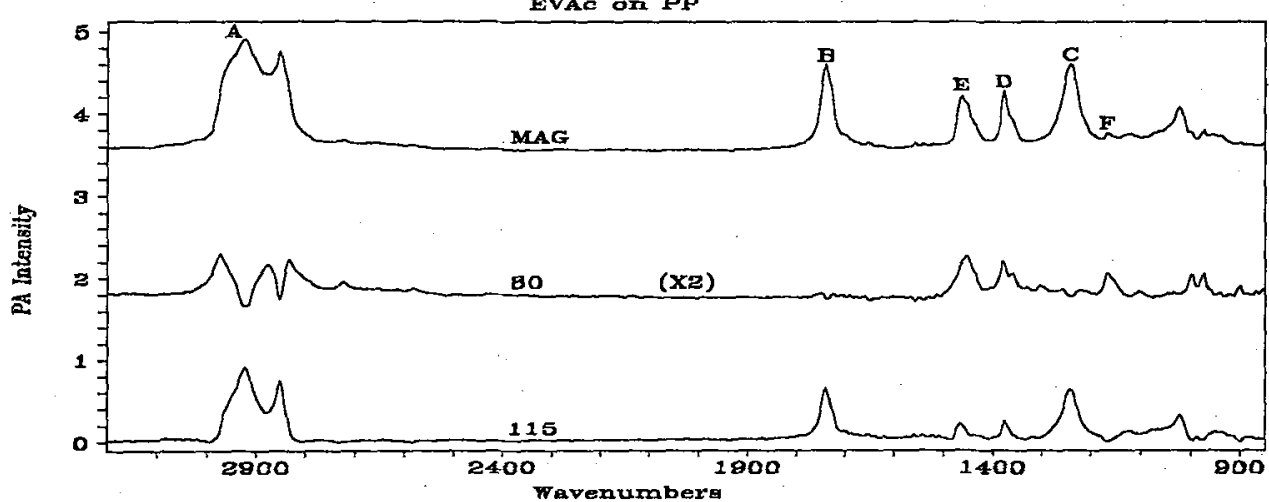

(b)

พavenumbers

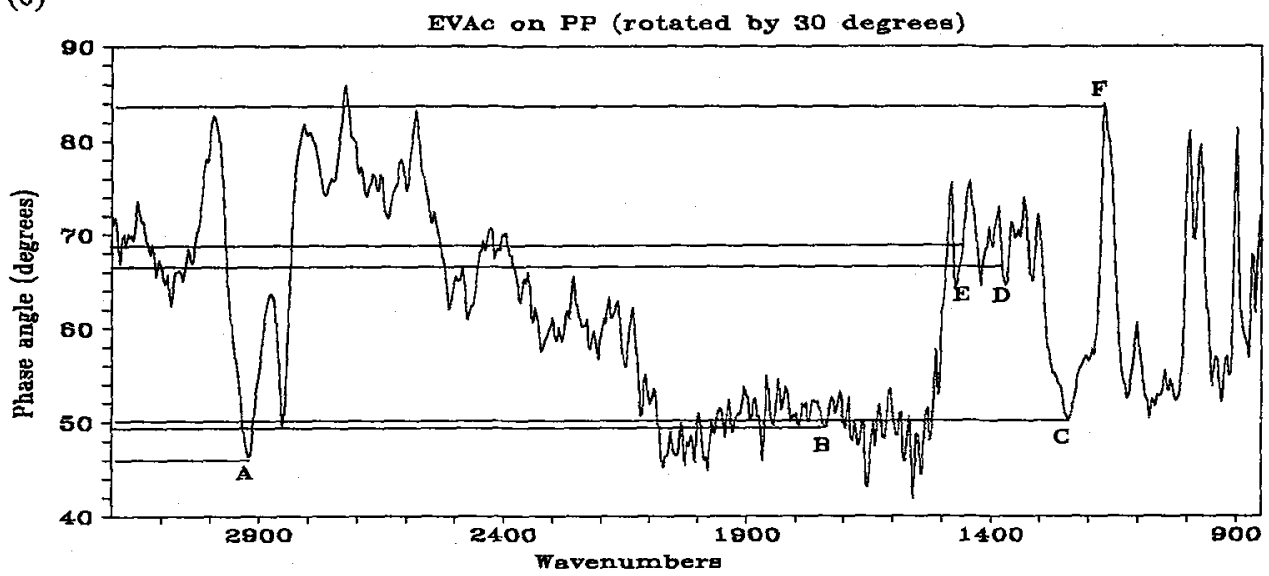

(c)

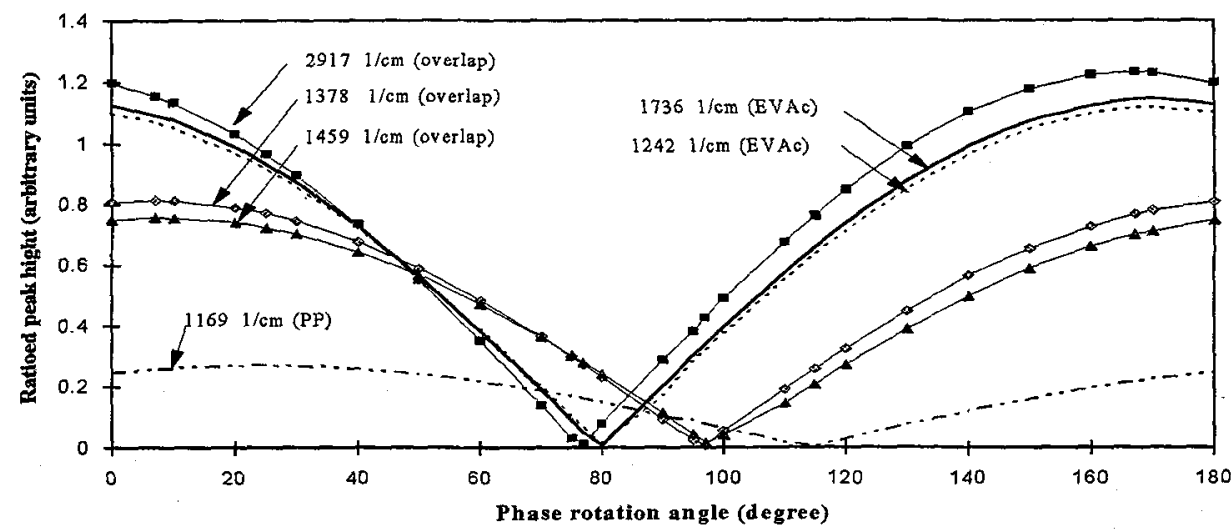

Figure 1. Ethylene vinyl acetate copolymer (EVAc, $12 \mu \mathrm{m}$ ) on polypropylene (PP, $60 \mu \mathrm{m}$ ). (a) PA magnitude spectrum and vector components at relative phase angles of $80^{\circ}$ and $115^{\circ}$. (b) PA phase spectrum. (c) Intensity variation of characteristic PA bands at different phase rotation angles. 
Table 1. EVAc on PP PA phase difference obtained from phase spectrum and phase rotation.

\begin{tabular}{ccccccc}
\hline Peak $\left(\mathrm{cm}^{-1}\right)$ & Assignment & Absorber(s) & PA Mag. & $\Phi$ spec. & $\Delta \Phi_{\mathrm{I}-\mathrm{A}}$ (spec.) & $\Delta \Phi_{\mathrm{I}-\mathrm{A}}$ (rot.) \\
A $(2917)$ & C-H stretch & EVAc/PP & 1.22 & $45.5^{\circ}$ & $0.0^{\circ}$ & $0^{\circ}$ \\
B $(1736)$ & C=O stretch & EVAc & 1.15 & $49.6^{\circ}$ & $4.1^{\circ}$ & $3^{\circ}$ \\
C $(1242)$ & C-O-C stretch & EVAc & 1.12 & $50.4^{\circ}$ & $4.9^{\circ}$ & $3^{\circ}$ \\
D $(1378)$ & C-H bend & EVAc/PP & 0.81 & $66.4^{\circ}$ & $20.9^{\circ}$ & $20^{\circ}$ \\
E $(1459)$ & C-H bend & EVAc/PP & 0.75 & $68.8^{\circ}$ & $23.3^{\circ}$ & $20^{\circ}$ \\
F $(1169)$ & C-C stretch & PP & 0.26 & $83.9^{\circ}$ & $38.4^{\circ}$ & $38^{\circ}$ \\
\hline
\end{tabular}

Table 2. PP on EVAc PA phase difference obtained from phase spectrum and phase rotation.

\begin{tabular}{ccccccc}
\hline Peak $\left(\mathrm{cm}^{-1}\right)$ & Assignment & Absorber(s) & PA Mag. & Фspec. & $\Delta \Phi$ I-A(spec.) & $\Delta \Phi$ I-A(rot.) \\
A $(2917)$ & C-H stretch & PP/EVAc & 2.9 & $50.7^{\circ}$ & $0.0^{\circ}$ & $0^{\circ}$ \\
D (1378) & C-H bend & PP/EVAc & 2.5 & $61.2^{\circ}$ & $10.6^{\circ}$ & $10^{\circ}$ \\
E (1459) & C-H bend & PP/EVAc & 2.4 & $63.8^{\circ}$ & $13.2^{\circ}$ & $13^{\circ}$ \\
F $(1169)$ & C-C stretch & PP & 1.0 & $81.4^{\circ}$ & $30.8^{\circ}$ & $30^{\circ}$ \\
B (1736) & C=O stretch & EVAc & 0.3 & $13.0^{\circ}$ & $-37.7^{\circ}$ & $-40^{\circ}$ \\
C $(1242)$ & C-O-C stretch & EVAc & 0.3 & $33.2^{\circ}$ & $-17.3^{\circ}$ & $-20^{\circ}$ \\
\hline
\end{tabular}

Table 3. Teflon ${ }^{\mathrm{TM}}$ Film on adhesive on backing laminate sample PA phase difference obtained from phase spectrum and phase rotation.

\begin{tabular}{lcccccc}
\hline Peak $\left(\mathrm{cm}^{-1}\right)$ & Assignment & Absorber(s) & PA Mag. & Фspec. & $\Delta \Phi$ I-A(spec.) & $\Delta \Phi$ I-A(rot.) \\
A (1250) & C-F stretch & Teflon & $1.34^{\circ}$ & $85.3^{\circ}$ & $0.0^{\circ}$ & $0^{\circ}$ \\
B (984) & C-C stretch & Teflon & $0.34^{\circ}$ & $55.4^{\circ}$ & $28.1^{\circ}$ & $29^{\circ}$ \\
C (1732) & C=O stretch & Adhesive & $0.33^{\circ}$ & $34.0^{\circ}$ & $49.5^{\circ}$ & $51^{\circ}$ \\
D (2960) & C-H stretch & Backing & $0.27^{\circ}$ & $11.3^{\circ}$ & $72.2^{\circ}$ & $73^{\circ}$ \\
\hline
\end{tabular}

Note: $\Delta \Phi_{\mathrm{A}-\mathrm{I}}=\Phi_{\mathrm{A}}-\Phi_{\mathrm{I}}$ and $\mathrm{I}=\mathrm{A}, \mathrm{B}, \mathrm{C}, \mathrm{D}, \mathrm{E}, \mathrm{F}$.
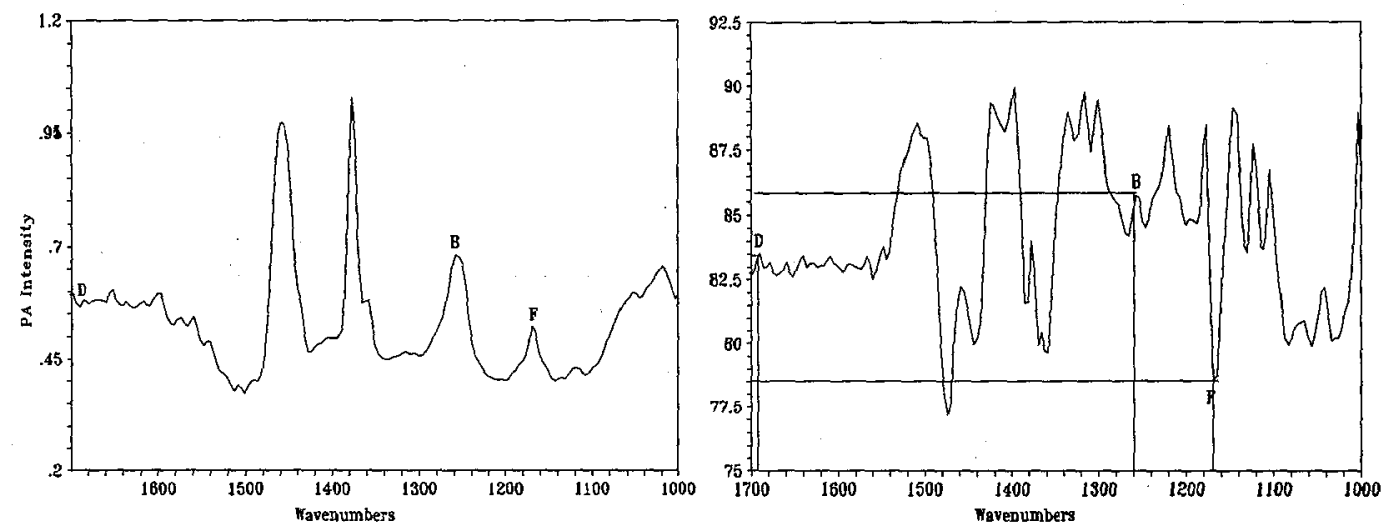

Figure 2. PA magnitude and phase spectra of HMDSiN $(0.7 \mu \mathrm{m})$ on CoTPP $(1.0 \mu \mathrm{m})$ on PP $(60 \mu \mathrm{m})$ collected at modulation frequency of $200 \mathrm{~Hz}$. 
Table 2 summarizes data from an experiment exactly parallel to the one reported in Figure 1 and Table 1. In this experiment the sample was simply turned over so that the $60 \mu \mathrm{m}$ of polypropylene was on top, that is, the sample was illuminated from the PP side and the $12.5 \mu \mathrm{m}$ of EVAc became the "substrate". In these data the bands of the EVAc (A, B, and C) are very weak in intensity, but nevertheless detectable, occurring as they do in regions where the PP is transparent. The phase difference between the signals from (A) the strongest PP band (overlapping, but because of the thickness of the PP layer, now completely obscuring the EVAc band), and that of (B) the strongest of the EVAc bands, is $37.7^{\circ}\left(140^{\circ}\right)$. Table 3 summarizes data from an experiment similar to those reported above in which the sample is composed of three layers, $12 \mu \mathrm{m}$ of Teflon ${ }^{\mathrm{TM}}$ on top of the adhesive and backing of a pressure sensitive tape. In these data phase differences between the saturated C-F stretching band (A), which can be taken to indicate the surface phase, and that of the $\mathrm{C}-\mathrm{O}$ stretch from the middle adhesive layer and the $\mathrm{C}-\mathrm{H}$ stretch from the underlying backing, are $49.5^{\circ}$ and $72.2^{\circ}$, respectively. The phase of the weak Teflon ${ }^{\mathrm{TM}}$ band $(\mathrm{B})$ is also observed.

Figure 3 shows the magnitude and phase spectra of a sample consisting of two consecutive plasma polymer surface layers of about $1 \mu \mathrm{m}$ thickness on a $60 \mu \mathrm{m}$ polypropylene substrate. The phase differences between signals at $1260 \mathrm{~cm}^{-1}$ (hexamethyldisilazane-based plasma polymer, HMDSiN), 1690 $\mathrm{cm}^{-1}$ (cobalt tetraphenylporphyrin-based plasma polymer, CoTPP) and $1170 \mathrm{~cm}^{-1}$ (polypropylene, PP) are distinct and reproducible to $\pm 0.5^{\circ}$.

These data illustrate the qualitative use of the step-scan FT-IR PAS phase in spectral depth profiling of several laminar polymeric materials. The important aspects of the results to note are: (1) that the use of the vibrational spectrum (as compared to the electronic spectrum) provides narrow, distinctive and non-overlapping absorption bands for identification of components; (2) that the use of FT-IR allows the use of a wide spectral range and thus access to the complete selection of spectral bands; (3) that the step-scan mode provides easy extraction of the PA signal phase data; (4) that the phase spectra in general give better and more useful phase distinction, and thus depth distinction, than the use of the simple phase rotation method; (5) that sub-surface components in laminar materials can be detected and their spectra selected out by phase beneath overlayers much thicker than the formal thermal diffusion length in regions where surface layers are transparent; and (6) that surface layers on the order of $1 \mu \mathrm{m}$ thick can be distinguished and their sequence determined by use of the step-scan FT-IR phase spectrum.

\section{REFERENCES}

[1] R. E. Imhof, D. J. S. Birch, F. R. Thornley, J. R. Gilchrist and T. A. Strivens, J. Phys. E: Sci. Instrum. 17, 521 (1984).

[2] J. W. Childers, R. Rhöl and R. A. Palmer, Anal. Chem.58, 2629 (1986).

[3] C. K.N. Patel and A. C. Tam, Appl. Phys Lett. 34, 467 (1979) and 36, 7 (1980).

[4] J. A. Graham, W. M. Grim, III and W. G. Fateley, in Eds., J. R. Ferraro and L. J. Basile, "Fourier Transform Infrared Spectroscopy", Vol. 4, Academic Press, Orlando, 1985.

[5] D. G. Mead, S. R. Lowery, D. W. Vidrine and D. R. Mattson, Proc. 4th Int. Conf. Inf rared Millimeter Waves, p 231 (1979) and M. G. Rockley, Chem. Phys. Lett. 68, 455 (1979).

[6] J. F. McClelland, S. Luo, R. W. Jones and L. M. Seaverson, Springer Ser.Opt.Sci. 69, 113(1992).

[7] L. B. Lloyd, S. M. Riseman, R. K. Burnham, E. M. Eyring and M. M. Farrow, Rev. Sci. Instrum. 51, 1488 (1980).

[8] D. Débarre, A. C. Boccara, and D. Fournier, Appl. Opt. 20, 4281 (1981).

[9] C. J. Manning, R. M. Dittmar, J. L. Chao and R. A. Palmer, Infrared Physics 33, 53 (1992).

[10] R. M. Dittmar, J. L. Chao and R. A. Palmer, Appl. Spectrosc. 45, 1104 (1991); R. A. Palmer and R. M. Dittmar, Thin Solid Films, Section A 223, 31 (1993).

[11] J. Chamberlain, Infrared Phys. 11, 25 (1971).

[12] A. Simon, 6th International Conference on Fourier Transform Spectroscopy, August, 1987, Vienna.

[13] M. J. Smith, C. J. Manning, R. A. Palmer, and J. L. Chao, Appl. Spectrosc. 42, 546 (1988).

[14] R. A. Crocombe, R. Curbelo, J. Leonardi and D. B. Johnson, SPIE Proceedings, Vol. 1575, 189 (1991); R. A. Crocombe, S. V. Compton, and J. Leonardi, ibid. 193 (1991).

[15] A. Rosencwaig and A. Gersho, J. Appl. Phys. 47, 64 (1976).

[16] B. Mongeau, G. Rousset, and L. Bertrand, Can. J. Phys. 64, 1056 (1986).

[17] J. F. Power and M. C. Prystay, 8th Int. Topical Meeting on Photoacoustic and Photothermal Phenomena, 1994, Paper HP-12.

[18] C. J. Manning, R. A. Palmer and J. L. Chao, Rev. Sci. Instrum. 62, 1219 (1991). 